Mens

revue d'histoire intellectuelle de l'Amérique française

Michael D. Behiels. Canada's Francophone Minority

Communities. Constitutional Renewal and the Winning of

School Governance. Montréal et Kingston, McGill-Queen's

University Press, 2004. 438 p.

\title{
Yves Frenette
}

Volume 8, numéro 1, automne 2007

URI : https://id.erudit.org/iderudit/1023154ar

DOI : https://doi.org/10.7202/1023154ar

Aller au sommaire du numéro

Éditeur(s)

Centre de recherche en civilisation canadienne-française

ISSN

1492-8647 (imprimé)

1927-9299 (numérique)

Découvrir la revue

Citer ce compte rendu

Frenette, Y. (2007). Compte rendu de [Michael D. Behiels. Canada's Francophone Minority Communities. Constitutional Renewal and the Winning of School

Governance. Montréal et Kingston, McGill-Queen's University Press, 2004.

438 p.] Mens, 8(1), 148-151. https://doi.org/10.7202/1023154ar 
l'auteur pour expliquer l'ostracisme qui a frappé Rodolphe Mathieu et son œuvre étant en grande partie fondée sur l'affrontement entre le clérico-nationalisme et le modernisme, l'argument est fragilisé par ce manque de précision et de profondeur dans l'analyse des idées et des esthétiques musicales.

Ajoutons finalement que le livre aurait été enrichi par l'ajout d'un disque comprenant quelques pièces de Rodolphe Mathieu et de compositeurs contemporains ayant adopté un style différent du sien. Si les spécialistes de la musique connaissent sans doute les œuvres de Mathieu et des autres compositeurs de son époque, il n'en va pas de même pour tous. Il serait plus aisé pour les néophytes de comprendre en quoi consistait l'originalité et la modernité de la musique de Rodolphe Mathieu en écoutant quelques-unes de ses compositions et en les comparant avec des œuvres d'inspiration plus traditionnelle.

Caroline Durand Département d'bistoire Université McGill

\begin{abstract}
Michael D. Behiels. Canada's Francophone Minority Communities. Constitutional Renewal and the Winning of School Governance. Montréal et Kingston, McGill-Queen's University Press, 2004. $438 \mathrm{p}$.
\end{abstract}

Dans cette monographie, Michael Behiels raconte l'histoire de la conquête de la gouvernance scolaire par les minorités francophones du Canada dans les deux dernières décennies du $\mathrm{XX}^{\mathrm{e}}$ siècle en faisant une lecture serrée des sources disponibles. Il est rare que les historiens s'aventurent sur le 
terrain du passé très récent et il faut savoir gré à Behiels de l'avoir fait. Son livre sera très utile, non seulement aux spécialistes des communautés de langue officielle, mais aussi à quiconque s'intéresse à l'évolution constitutionnelle du Canada.

Dans ses travaux antérieurs, Behiels nous a habitués à une écriture engagée en faveur d'une conception fédéraliste de l'histoire canadienne et son dernier livre ne déçoit pas à cet égard, comme en témoigne le choix de son préfacier, le sénateur Serge Joyal. La recherche ayant mené à la rédaction du livre est exhaustive et la narration descriptive de Behiels colle aux sources ; l'auteur ne peut toutefois s'empêcher d'insérer de brefs commentaires qui témoignent de ses partis pris. Contrairement à ce qu'on pourrait penser, ses héros ne sont pas les hommes politiques libéraux, Pierre Elliott Trudeau en tête, mais plutôt les chefs de file franco-ontariens, francoalbertains et franco-manitobains, qui, contre vents et marées, contre les intérêts des divers gouvernements provinciaux, y compris bien sûr celui du Québec, luttent pour obtenir des écoles et des conseils scolaires contrôlés par les francophones. Ce faisant, ils se transforment en défenseurs acharnés d'une conception bilingue et biculturelle du Canada, et ils sont au premier plan de la démocratisation de la politique constitutionnelle canadienne. Behiels montre également beaucoup de sympathie pour les intellectuels qui fournissent des munitions aux combattants minoritaires, en particulier le juriste Michel Bastarache.

La narration descriptive de Behiels est toute en nuances. La complexité du processus constitutionnel, l'impact de la conjoncture politique, tant nationale que provinciale, les enjeux divers, les divisions entre les minorités et au sein de chaque minorité, constituent autant d'éléments qui influencent le débat. 
Avant d'aborder l'étude de la conquête de la gouvernance scolaire proprement dite, Behiels se penche dans un premier chapitre sur la renaissance des minorités francophones pendant les décennies 1960 et 1970, renaissance qui prend place grâce à l'arrivée au pouvoir d'une nouvelle génération de leaders résolument "modernes" et grâce à la Loi sur les langues officielles (1969). Avec l'encouragement d'Ottawa, ces chefs de file fondent la Fédération des francophones hors Québec en 1975 et développent leur propre vision constitutionnelle. S'appuyant sur les travaux disponibles, l'analyse de Behiels est compétente, mais elle met beaucoup trop l'accent sur l'effet catalyseur de la Révolution tranquille aux dépens des facteurs endogènes de changement au sein des minorités depuis le tournant du XX' ${ }^{\mathrm{e}}$ siècle. Le chapitre 2 porte sur le rôle des associations provinciales dans le processus de révision de la constitution suite à la victoire du « Non » lors du référendum sur la souveraineté du Québec en 1980. Appuyés par le gouvernement Trudeau, les représentants des minorités réussissent à inscrire leurs préoccupations dans la Charte des droits et libertés de 1982. Pour utiliser l'expression de l'auteur, les chefs de file apprennent rapidement à maitriser les règles du jeu de la "politique méga-constitutionnelle ». Les chapitres 3 à 5 constituent le cœur de l'ouvrage. Behiels s'y penche sur les cas des francophones de l'Ontario, de l'Alberta et du Manitoba ; ceux-ci font des pressions politiques et judiciaires pour obtenir la gouvernance scolaire face à des gouvernements provinciaux hostiles et très habiles à exploiter les divisions de toutes sortes au sein des communautés. Dans les deux chapitres suivants, l'auteur étudie la position des francophones hors-Québec dans les débats entourant les accords du Lac Meech et de Charlottetown entre 1987 et 1992, ainsi que les hésitations et déchirures au sein du leadership, notamment entre les Acadiens et les Franco- 
Albertains. Ici, les gains des minorités francophones sont modestes, en raison de la combination de forces externes négatives : «The future of Canada's official language minorities was sacrificed on the altar of Québécois nationalism, expansive provincial rights, and Ottawa's perceived need to head off a clash with Canada's highly nationalistic and politicized aboriginal communities (p. 323). », commente Behiels.

Il ressort de tout cela que les leaders francophones sont devenus politiquement matures très rapidement et que, contrairement à des représentations qui persistent, ils n'ont nullement été les marionnettes du gouvernement fédéral. C'est par l'adoption de stratégies multiformes que ces hommes et ces femmes ont réussi à tirer leur épingle du jeu. Leur vision et leur stratégie n'étaient pas monolithiques parce qu'ils provenaient de communautés elles-mêmes diversifiées, sinon fragmentées géographiquement, socialement, culturellement, linguistiquement et politiquement.

En terminant, déplorons le fait que la recherche exhaustive de Behiels nuit à sa stratégie discursive. Il reprend chaque document en détail, même si cela entraîne des redites fastidieuses. En conséquence, le lecteur perd souvent le fil de son argument, en dépit des conclusions de fin de chapitre. Et que dire des nombreuses erreurs typographiques dans les citations en français? Leur présence est ironique dans un ouvrage qui prône les bienfaits du bilinguisme.

Yves Frenette

Centre de recherche en civilisation canadienne-française Université d'Ottawa 